\title{
Pancreas-Targeted NIR Fluorophores for Dual-Channel Image-Guided Abdominal Surgery
}

\author{
Hideyuki Wada1,2, Hoon Hyun ${ }^{1}$, Christina Vargas ${ }^{1,3}$, Julien Gravier ${ }^{1,4}$, GwangLi Park ${ }^{1}$, Sylvain Gioux ${ }^{1}$, John \\ V. Frangioni ${ }^{1,5,6}$, Maged Henary ${ }^{7 凶}$, and Hak Soo Choi ${ }^{1,8}$ \\ 1. Division of Hematology/Oncology, Department of Medicine, Beth Israel Deaconess Medical Center and Harvard Medical School, Bos- \\ ton, MA 02215. \\ 2. Department of Gastroenterological Surgery II, Hokkaido University Graduate School of Medicine, Sapporo 060-8638, Japan. \\ 3. Division of Plastic and Reconstructive Surgery, Department of Surgery, Beth Israel Deaconess Medical Center, Boston, MA 02215. \\ 4. INSERM, CRI, U823, Institut Albert Bonniot, 38042 Grenoble, France. \\ 5. Department of Radiology, Beth Israel Deaconess Medical Center and Harvard Medical School, Boston, MA 02215. \\ 6. Curadel, LLC, 377 Plantation Street, Worcester, MA 01605. \\ 7. Department of Chemistry, Center for Diagnostics and Therapeutics, Georgia State University, Atlanta, GA 30303. \\ 8. Department of Cogno-Mechatronics Engineering, Pusan National University, Busan 609-735, South Korea.
}

$\triangle$ Corresponding author: Hak Soo Choi, Ph.D. Tel: 617-667-6024; Email: hchoi@bidmc.harvard.edu or Maged Henary, Ph.D. Tel: 404-413-5566; Email: mhenary1@gsu.edu.

() Ivyspring International Publisher. This is an open-access article distributed under the terms of the Creative Commons License (http://creativecommons.org/ licenses/by-nc-nd/3.0/). Reproduction is permitted for personal, noncommercial use, provided that the article is in whole, unmodified, and properly cited.

Received: 2014.08.04; Accepted: 2014.09.10; Published: 2015.01.01

\begin{abstract}
Objective: Pancreas-related complications are some of the most serious ones in abdominal surgery. The goal of this study was to develop and validate novel near-infrared (NIR) fluorophores that would enable real-time pancreas imaging to avoid the intraoperative pancreatic injury.

Design: After initial screening of a large NIR fluorophore library, the performance of 3 selected pancreas-targeted $700 \mathrm{~nm}$ NIR fluorophores, T700-H, T700-F, and MB, were quantified in mice, rats, and pigs. Dose ranging using 25 and $100 \mathrm{nmol}$, and $2.5 \mu \mathrm{mol}$ of T700-F, and its imaging kinetics over a $4 \mathrm{~h}$ period were tested in each species. Three different $800 \mathrm{~nm}$ NIR fluorophores were employed for dual-channel FLARE TM imaging in pigs: $2 \mu \mathrm{mol}$ of ZW800-I for vessels and kidney, I $\mu \mathrm{mol}$ of ZW800-3C for lymph nodes, and $2 \mu \mathrm{mol}$ of ESNF3 I for adrenal glands.

Results: T700-F demonstrated the highest signal to background ratio (SBR), with peak SBR at $4 \mathrm{~h}$ postinjection in mice. In pigs, T700-F produced an SBR $\geq 2$ against muscle, spleen, and lymph nodes for up to $8 \mathrm{~h}$ after a single intravenous injection. The combination of T700-F with each $800 \mathrm{~nm}$ NIR fluorophore provided simultaneous dual-channel intraoperative imaging of pancreas with surrounding organs in real time.

Conclusion: Pancreas-targeted NIR fluorophores combined with the FLARE dual-channel imaging system enable the real-time intraoperative pancreas imaging which helps surgeons perform safer and more curative abdominal surgeries.
\end{abstract}

Key words: Pancreas-related complications, Postoperative pancreatic fistula, Intraoperative pancreatic injury, Image-guided surgery, Near-Infrared fluorescence.

\section{Introduction}

Pancreas-related complications in abdominal surgery are serious and sometimes fatal. Unrecognized intraoperative pancreatic injury results in many pancreas-related complications, such as postoperative pancreatic fistulae, peripancreatic abscesses, and pancreatitis $[1,2]$. These can progress to more serious complications such as intra-abdominal hemorrhage and septic shock, which result in longer hospital stays 
and higher medical costs [3]. The incidence of pancreas-related complications after gastrectomy with radical lymphadenectomy, which is the standard procedure in advanced gastric cancers, ranges from 5.8 to $49.7 \%$ [3-8]. Even performing laparoscopic gastrectomy, which offers a magnified view of the surgical field, reported complication rates range from 4.3 to $7.2 \%[1,2]$. In addition to other gastrointestinal surgeries such as colectomy and splenectomy [9], urological surgeries including nephrectomy and adrenalectomy also have a high risk factor of pancreas-related complications [10].

Intraoperative pancreatic injuries are typically caused by the proximity of the pancreas to surrounding organs and the difficulty in distinguishing the edge of the pancreas from surrounding fat tissue, especially in obese patients. Real-time, high sensitivity identification of the pancreas intraoperatively could help avoid such injury. Previously we reported that near-infrared (NIR) fluorescent light could be used to identify both the pancreas and insulinoma by employing the clinically available NIR fluorophore methylene blue (MB) [11]. Although MB provided contrast between the pancreas and surrounding organs, it is not an ideal NIR contrast agent because of low signal intensity, nonspecific uptake, and short retention time in the pancreas.

Recently, we developed a library of NIR fluorophores targeted to various tissues and organs including thyroid and parathyroid [12], lymph nodes [13], nerves [14], adrenal glands [15], and bone [16] with high signal in the target and relatively low background after a single intravenous injection. Furthermore, screening the in vivo performance of our over 300 novel compounds, we found T700-F and T700-H could target the pancreas specifically. Combined with our FLARE intraoperative imaging system, which provides simultaneous dual-channel NIR fluorescence imaging along with color video, various surgeries can now be performed under real-time image guidance [17]. In this study, we hypothesized that the same approach could be used for pancreas imaging, and focused our experiments on novel agents that could outperform MB.

\section{Materials and Methods}

NIR Fluorescent Contrast Agents: Methylene blue (MB; USP $1 \%, 10 \mathrm{mg} / \mathrm{mL}$ ) was purchased from Taylor Pharmaceuticals (Decatur, IL). T700-H and T700-F were synthesized as described in our previous reports [12,18-20], and served as pancreas targeting $700 \mathrm{~nm}$ emitting NIR fluorophores. Three different $800 \mathrm{~nm}$ NIR fluorophores including ZW800-1, ZW800-3C, and ESNF31, were also used for the dual channel imaging of surrounding tissue: ZW800-1 for
NIR angiography and kidney imaging [21]; ZW800-3C for PLN imaging [13], and ESNF31 for adrenal glands [15]. MB was used as purchased without further treatment $(31.3 \mathrm{mM})$, and all other NIR fluorophores were dissolved in dimethyl sulfoxide (DMSO) as a $10 \mathrm{mM}$ stock solution.

Optical Property Measurements: Optical properties of NIR fluorescent contrast agents were measured in 100\% fetal bovine serum (FBS) supplemented with $50 \mathrm{mM}$ HEPES, $\mathrm{pH}$ 7.4. The quantum yield (QY) of $700 \mathrm{~nm}$ NIR fluorophores was measured using Oxazine 725 (Sigma-Aldrich) in ethylene glycol (QY = $19 \%$ ) was used as a calibration standard under conditions of matched absorbance at $655 \mathrm{~nm}$ [22], while indocyanine green (ICG) in DMSO (QY $=13 \%$ ) was used for the QY of $800 \mathrm{~nm}$ NIR fluorophores with a matched absorbance at $770 \mathrm{~nm}$ [23]. For in vitro optical property measurements, online fiberoptic HR2000 absorbance $(200-1100 \mathrm{~nm})$ and USB2000FL fluorescence (350-1000 nm) spectrometers (Ocean Optics, Dunedin, FL) were used. NIR excitation was provided by $5 \mathrm{~mW}$ of $655 \mathrm{~nm}$ red laser pointer (Opcom Inc., Xiamen, China) and $8 \mathrm{~mW}$ of $765 \mathrm{~nm}$ NIR laser diode light source (Electro Optical Components, Santa Rosa, CA) coupled through a $300 \mu \mathrm{m}$ core diameter, NA 0.22 fiber (Fiberguide Industries, Stirling, NJ). In silico calculations of the distribution coefficient $(\log \mathrm{D}$ at $\mathrm{pH}$ 7.4) and 3D minimized structures were calculated using Marvin and JChem calculator plugins (ChemAxon, Budapest, Hungary).

Animal Models: Animals were housed in an Association for Assessment and Accreditation of Laboratory Animal Care (AAALAC)-certified facility and were studied under the supervision of Beth Israel Deaconess Medical Center's Institutional Animal Care and Use Committee (IACUC) in accordance with approved institutional protocols \#101-2011 for rodents and \#034-2013 for pigs. Male CD-1 mice $(n=42)$ averaging $21 \mathrm{~g}$ and male Sprague-Dawley rats $(\mathrm{n}=6)$ averaging $262 \mathrm{~g}$ (Charles River Laboratories, Wilmington, MA) were anaesthetized with $100 \mathrm{mg} / \mathrm{kg}$ ketamine and $10 \mathrm{mg} / \mathrm{kg}$ xylazine intraperitoneally (Webster Veterinary, Fort Devens, MA) and a midline laparotomy was performed to expose the viscera. Female Yorkshire pigs $(\mathrm{n}=12)$ averaging $35.5 \mathrm{~kg}$ (E.M. Parsons and Sons, Hadley, MA) were induced with $4.4 \mathrm{mg} / \mathrm{kg}$ intramuscular Telazol (Fort Dodge Laboratories, Fort Dodge, IA) and intubated; anesthesia was maintained with $2 \%$ isoflurane (Baxter Healthcare, Deerfield, IL). Following anesthesia, a $14-G$ central venous catheter was inserted into the external jugular vein, and saline was administered as needed. A midline laparotomy and a left trans-rectus incision were performed for satisfactory intraoperative imaging. Electrocardiogram, heart rate, pulse 
oximetry, and body temperature were monitored during the experiment.

NIR Fluorescence Imaging System: The dual-NIR channel FLARE imaging system has been described in detail previously $[17,24]$. In this study, 670 $\mathrm{nm}$ excitation and $760 \mathrm{~nm}$ excitation fluence rates used were 4.0 and $11.0 \mathrm{~mW} / \mathrm{cm}^{2}$, respectively, with white light (400 to $650 \mathrm{~nm}$ ) at 40,000 lx. Color image and 2 independent channels $(700 \mathrm{~nm}$ and $800 \mathrm{~nm})$ of NIR fluorescence images were acquired simultaneously with custom software at rates up to $15 \mathrm{~Hz}$ over a 15-cm diameter field of view (FOV). In the color-NIR merged images, $700 \mathrm{~nm}$ NIR fluorescence and $800 \mathrm{~nm}$ fluorescence were pseudo-colored red and lime green, respectively. The imaging system was positioned at a distance of 18 inches from the surgical field. Camera exposure time and normalization were held constant for each experiment.

Intraoperative Pancreas Imaging in Mice, Rats, and Pigs: For initial in vivo screening, $25 \mathrm{nmol}$ of T700-H and T700-F were injected intravenously into CD-1 mice $4 \mathrm{~h}$ prior to imaging $(\mathrm{n}=3$ per each fluorophore). As a control, $120 \mathrm{nmol}(1.5 \mathrm{mg} / \mathrm{kg})$ of MB was injected 15 min prior to imaging $(n=3)$ [11]. In the dose optimization study, we injected 5 different doses of T700-F (0, 10, 25, 50, and $100 \mathrm{nmol})$ and images were taken at $4 \mathrm{~h}$ post-injection $(\mathrm{n}=3$ per each dose). For the kinetics study, we injected $25 \mathrm{nmol}$ of T700-F intravenously and observed the fluorescent signal of pancreas at 6 different time points $(0,1,2,4$, 8 , and $24 \mathrm{~h})(\mathrm{n}=3$ per each time point). For rat studies, $1.2 \mu \mathrm{mol}$ of MB $(1.5 \mathrm{mg} / \mathrm{kg})$ and $100 \mathrm{nmol}$ of T700-F were injected intravenously $15 \mathrm{~min}$ and $4 \mathrm{~h}$ prior to imaging, respectively ( $\mathrm{n}=3$ each NIR fluorophore). All NIR fluorophores except for MB were diluted into $5 \%$ dextrose in water (D5W) before injection, and 100 $\mu \mathrm{L}$ was injected into mice and $500 \mu \mathrm{L}$ was injected into rats, respectively.

For large animal swine studies, $164 \mu \mathrm{mol}(1.5$ $\mathrm{mg} / \mathrm{kg}$ ) of $\mathrm{MB}$ and $2.5 \mu \mathrm{mol}$ of T700-F were injected intravenously. We observed the fluorescent signals of viscera for $1 \mathrm{~h}$ post-injection, calculated a signal-to-background ratio (SBR) at 6 different time points $(0,3,5,15,30$, and $60 \mathrm{~min})$, and compared the performance ( $\mathrm{n}=3$ per each fluorophore). Next, we performed kinetics study using $2.5 \mu \mathrm{mol}$ of T700F, and images were recorded at 11 different time points $(0,15,30,60,90,120,180,240,300,360$, and 480) $(\mathrm{n}=$ $3)$. Finally, we tested dual-channel imaging of pancreas with surrounding vital tissues and organs including arteries, kidneys, pan lymph nodes (PLNs), and adrenal glands: $2 \mu \mathrm{mol}$ of ZW800-1 for NIR arteriography and kidney imaging, $1 \mu \mathrm{mol}$ of ZW800-3C for PLN imaging, and $2 \mu \mathrm{mol}$ of ESNF31 for adrenal gland imaging ( $\mathrm{n}=1$ for each study). The NIR angi- ography was recorded immediately after a single intravenous injection of ZW800-1 and kidneys were imaged $2 \mathrm{~h}$ post-injection. The PLN imaging was performed $4 \mathrm{~h}$ post-injection, while adrenal glands were imaged at $30 \mathrm{~min}$ post-injection. These imaging times were chosen based on prior optimization $[13,15,25]$. Each NIR agent was diluted into $10 \mathrm{~mL}$ of D5W prior to injection.

NIR Fluorescence Microscopy: For histological evaluations, pancreatic tissues were resected from rats after scheduled imaging using $\mathrm{MB}$ and T700-F, embedded in Tissue-Tek OCT compound (Fisher Scientific, Pittsburgh, PA), and flash frozen in liquid nitrogen. Tissue was cryosectioned at $10 \mu \mathrm{m}$ intervals and observed using a NIR fluorescence microscope. Consecutive sections were stained with hematoxylin and eosin (H\&E). NIR fluorescence microscopy was performed on a 4 filter-set Nikon Eclipse TE300 epifluorescence microscope to confirm the fluorescence of the pancreas as previously described [26,27]. The microscope was equipped with a $100 \mathrm{~W}$ mercury light source, NIR-compatible optics, and a NIR-compatible 4X, 10X, 20X, and 40X Plan Fluor objective lens (Nikon, Melville, NY). Custom filter sets (Chroma Technology Corporation, Brattleboro, VT) composed of a $650 \pm 22 \mathrm{~nm}$ excitation filter and a $710 \pm$ $25 \mathrm{~nm}$ emission filter were used to detect the pancreas fluorescence signal. Images were acquired on an Orca-AG (Hamamatsu, Bridgewater, NJ) and QImaging 12-bit camera for color imaging (Surrey, BC, Canada). Image acquisition and analysis was performed using iVision software (BioVision Technologies, Exton, PA).

Quantitation and Statistical Analysis: The fluorescent intensity (FI) of a region of interest (ROI) over the pancreas, rectus abdominis muscle, liver, spleen, duodenum, lymph nodes, and kidneys were quantified using custom FLARE software. The performance metric for this study was SBR. SBR = FI of ROI / background (BG) intensity. The rectus abdominis muscles, liver, spleen, duodenum, lymph nodes, and kidneys were used as BG compared to the signal in the pancreas to yield pancreas-to-muscle ratio $(\mathrm{Pa} / \mathrm{Mu})$, pancreas-to-liver ratio $(\mathrm{Pa} / \mathrm{Li})$, pancreas-to-spleen ratio $(\mathrm{Pa} / \mathrm{Sp})$, pancreas-to-duodenum ratio $(\mathrm{Pa} / \mathrm{Du})$, pancreas-to-lymph node ratio $(\mathrm{Pa} / \mathrm{LN})$, and pancreas-to-kidney ratio $(\mathrm{Pa} / \mathrm{Ki})$. At least 3 animals were analyzed for SBR at each time or dose point. Results were presented as mean \pm standard deviation (SD). The statistical analysis was performed using the Unpaired $T$ test between two groups and a one-way ANOVA followed by Tukey's multiple comparisons test between multiple groups. A $P$ value of less than 0.05 was considered significant: ${ }^{*} P<0.05$, ${ }^{* *} P<0.01$, and ${ }^{* * *} P<0.001$. 


\section{Results}

Optical properties of NIR fluorescent contrast agents. The chemical structure, absorbance, and fluorescence spectra for MB, T700-H, T700-F, ZW800-1, ZW800-3C, and ESNF31 are shown in Figure 1. Additionally, Table 1 details the optical properties of each NIR fluorophore in 100\% serum. T700 fluorophores have 2-3 fold higher extinction coefficient and more than 3-fold higher QY compared to $M B$, which resulted in a total of 8-fold higher molecular brightness (Table 1). To summarize, MB, T700-H, and T700-F exhibited optical properties compatible with NIR fluorescence channel \#1 (700 $\mathrm{nm})$ of the FLARE imaging system, while ZW800-1, ZW800-3C, and ESNF31 were imaged using NIR fluorescence channel \#2 (800 nm), which permits simultaneous, real-time, dual-channel intraoperative imaging.
A.

MB

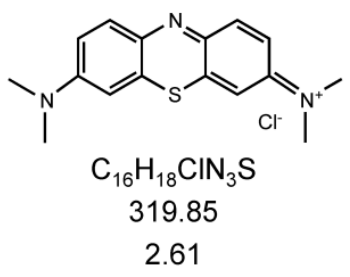

MW (Da):

$\log D, \mathrm{pH} 7.4$ :

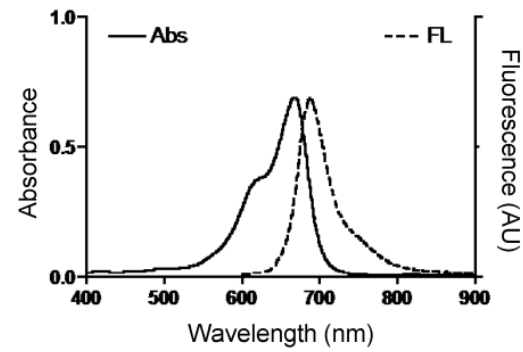

B.
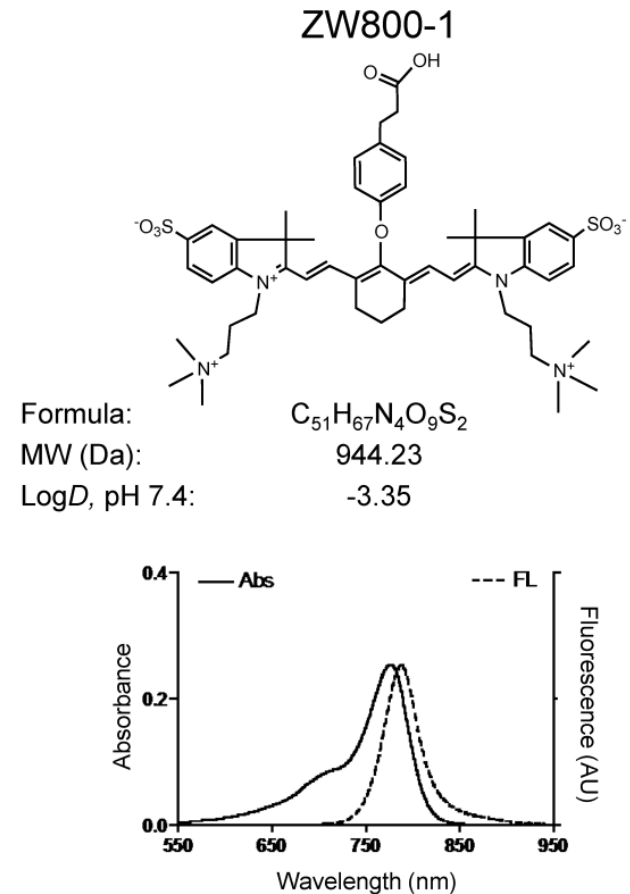

\section{$700 \mathrm{~nm}$ NIR Fluorophores}

T700-H

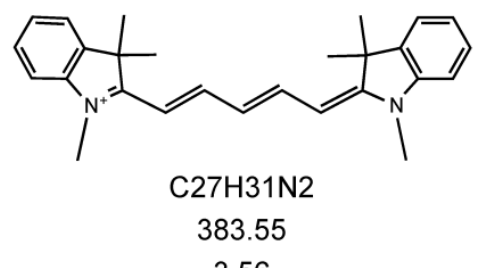

3.56

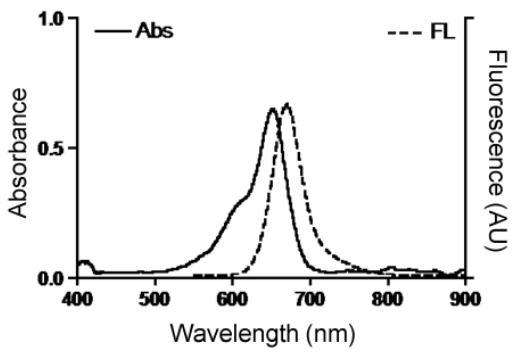

$\underline{800 \mathrm{~nm} \text { NIR Fluorophores }}$

ZW800-3C

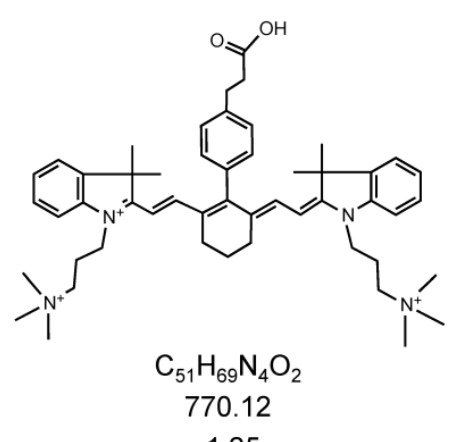

$-1.25$

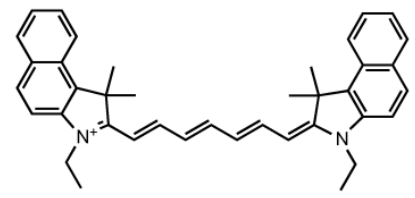

$\mathrm{C}_{39} \mathrm{H}_{41} \mathrm{~N}_{2}$

537.76

6.78

T700-F
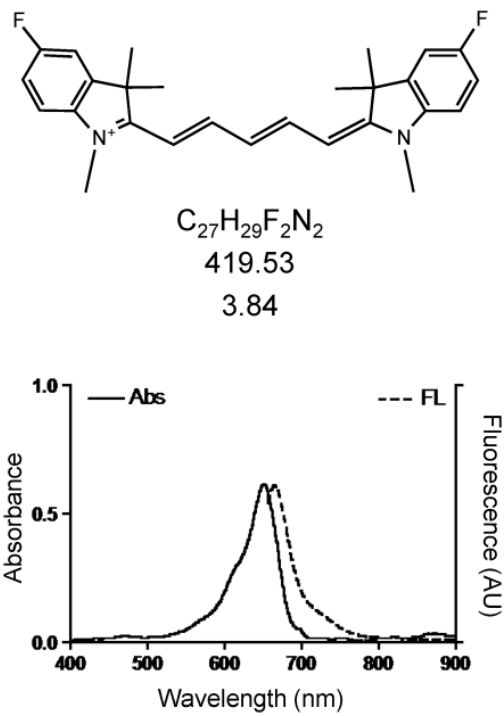

ESNF31
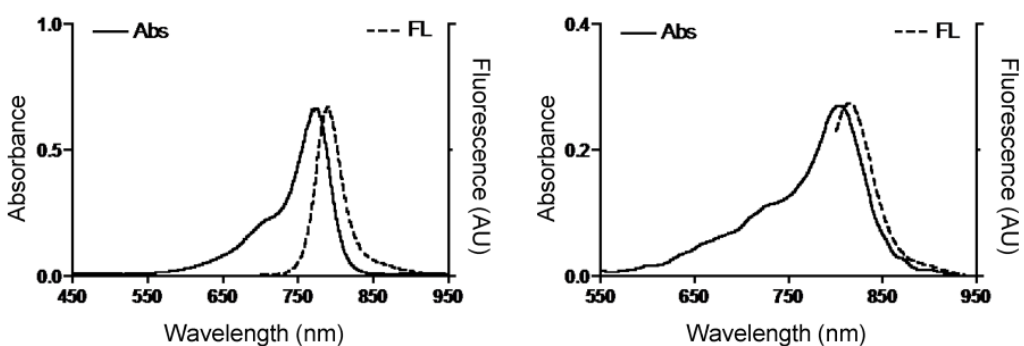

Figure I. Chemical Structure and Optical Properties of NIR Fluorophores. Chemical structure, molecular weight (MW), and logD at pH 7.4 were depicted using MarvinSketch (ChemAxon). Absorbance (Abs) and fluorescence (FL) emission spectra were measured in I00\% fetal bovine serum at $\mathrm{pH} 7.4$. a) $700 \mathrm{~nm} \mathrm{NIR} \mathrm{Fluorophores.} \mathrm{b)} 800 \mathrm{~nm} \mathrm{NIR}$ Fluorophores. 
Table I. Optical properties of NIR fluorophores in 100\% serum supplemented with $50 \mathrm{mM} \mathrm{HEPES} \mathrm{(pH} \mathrm{7.4).}$

\begin{tabular}{|c|c|c|c|c|c|c|}
\hline Optical Property & MB & $\mathrm{T} 700-\mathrm{H}$ & $\mathrm{T} 700-\mathrm{F}$ & ZW800-1 & ZW800-3C & ESNF31 \\
\hline FLARE Imaging Channel & \multicolumn{3}{|c|}{700 nm NIR (Channel \#1) } & \multicolumn{3}{|c|}{800 nm NIR (Channel \#2) } \\
\hline Extinction Coefficient $\left(\mathrm{M}^{-1} \mathrm{~cm}^{-1}\right)$ & 49,500 & 147,000 & 123,000 & 249,000 & 309,000 & 135,000 \\
\hline Absorbance Maximum (nm) & 665 & 648 & 651 & 772 & 774 & 803 \\
\hline Emission Maximum (nm) & 688 & 670 & 665 & 788 & 789 & 814 \\
\hline Quantum Yield (\%) & 9.6 & 30.4 & 29.9 & 15.1 & 16.1 & 10.8 \\
\hline Molecular Brightness $\left(\mathrm{M}^{-1} \mathrm{~cm}^{-1}\right)$ & 4,752 & 44,688 & 36,777 & 37,599 & 49,749 & 14,580 \\
\hline
\end{tabular}

In silico calculations of $\log \mathrm{D}$ at $\mathrm{pH} 7.4$ and total polar surface area were calculated using Marvin and JChem calculator plugins (ChemAxon, Budapest, Hungary).

Initial in vivo screening in mice. Our NIR fluorophore library consists of over 300 novel compounds. Initial screening in mice revealed 2 pentamethine indocyanine fluorophores, $\mathrm{T} 700-\mathrm{H}$ and T700-F, with unusually high uptake in pancreas (Supplementary Material: Figure S1). T700-F showed a stronger pancreas signal and higher contrast to surrounding organs, such as duodenum, spleen, and liver. Both, however, also had strong kidney signal due to renal elimination. We then compared these two NIR fluorophores to clinically available MB in mice and re-measured SBR. As shown Figure 2A, T700-F showed the highest uptake in pancreas compared to others, and a significant improvement in SBR was found when compared with $\mathrm{MB}$, which was injected at a 4-fold higher dose and exhibited a 10-fold shorter retention time [11]. Because T700-F demonstrated the highest SBR, we explored its dose optimization and kinetics, and expanded the study to 2 additional species, rats and pigs.

Kinetics and dose optimization of T700-F in mice. To optimize the best imaging time for pancreas (Pa), we injected $25 \mathrm{nmol}$ of T700-F intravenously into CD-1 mice and measured the SBR of pancreas and surrounding tissues over the course of $24 \mathrm{~h}$. As shown in Figure 2B, the highest SBR relative to liver ( $\mathrm{Li}$ ), spleen (Sp), and duodenum (Du), was achieved at $4 \mathrm{~h}$ post-injection although the background signal decreased continuously over time, the fluorescence signal of pancreas also diminished significantly after $4 \mathrm{~h}$ (Figure 2D). Therefore, dose optimization was performed at $4 \mathrm{~h}$ post-injection. Of note, no adverse reactions were encountered over the $24 \mathrm{~h}$ observation period.

For dose-optimization, we injected 5 different doses of T700-F (0, 10, 25, 50, and $100 \mathrm{nmol})$ into CD-1 mice, and images were acquired at $4 \mathrm{~h}$ post-injection. Signals in pancreas $(\mathrm{Pa})$ increased proportionally to dose, but background signal in surrounding organs, including spleen $(\mathrm{Sp})$ and duodenum $(\mathrm{Du})$, rose significantly above the $25 \mathrm{nmol}$ dose (Figure 2D). There was no significant difference in SBR of $(\mathrm{Pa} / \mathrm{Sp})$ and $(\mathrm{Pa} / \mathrm{Du}$ ) among 25 - $100 \mathrm{nmol}$ groups (Figure 2C).
Intraoperative pancreas imaging and histology in rats. We compared T700-F with MB in SD rats by injecting $100 \mathrm{nmol}$ of T700-F and $1.2 \mu \mathrm{mol}(1.5 \mathrm{mg} / \mathrm{kg})$ of $\mathrm{MB}$, respectively (Figure $3 \mathrm{~A}$ ). Although MB highlighted the pancreas immediately after a single intravenous injection, the fluorescent signal in pancreas diminished rapidly after $15 \mathrm{~min}$. Conversely, T700-F showed strong pancreas signal and low background uptake even at $4 \mathrm{~h}$ post-injection. After intraoperative imaging, we resected the pancreas and performed histological analysis (Figure 3B). NIR fluorescence microscopy confirmed $700 \mathrm{~nm}$ fluorescence signal in the resected pancreatic tissues. Both fluorophores accumulated in the intracellular space: T700-F stained both the exocrine pancreas (acini; AC) cells and islets of Langerhans (IL), while MB exhibited proportionally higher islet uptake.

In vivo pancreas imaging in pigs. To confirm that small animal results were not species-dependent, we performed pancreas imaging in $35 \mathrm{~kg}$ Yorkshire pigs using $164 \mu \mathrm{mol}(1.5 \mathrm{mg} / \mathrm{kg})$ of $\mathrm{MB}$ and $2.5 \mu \mathrm{mol}$ of T700-F. We administered more than a 65-fold higher dose of $\mathrm{MB}$, however, pancreatic contrast diminished rapidly over time while T700-F showed an extremely strong pancreas signal (Figure 4A). Although the initial uptake of T700-F in spleen was higher than in the pancreas, the fluorescence signal in spleen decreased gradually and was negligible at 60 min post-injection. Adequate SBR $(\mathrm{Pa} / \mathrm{Mu})$ of $\mathrm{MB}$ was only seen for the initial $15 \mathrm{~min}$ and the SBR decayed by $60 \mathrm{~min}$ to almost the pre-injection level (Figure 4B). In contrast, T700-F maintained its SBR $(\mathrm{Pa} / \mathrm{Mu})$ of $\approx$ 5.0 for $60 \mathrm{~min}$ without detectable decrease and there was a significant difference in SBR $(\mathrm{Pa} / \mathrm{Mu})$ for $1 \mathrm{~h}$ between $\mathrm{MB}$ and T700-F. We further investigated the kinetics of T700-F for up to $8 \mathrm{~h}$ by comparing signals in the pancreas $(\mathrm{Pa})$ with surrounding tissue and organs such as muscle $(\mathrm{Mu})$, spleen $(\mathrm{Sp})$, lymph nodes (Ln), and kidneys (Ki) (Figure 4C). Overall T700-F showed relatively high SBRs for up to $8 \mathrm{~h}$ without significant decrease in the pancreatic signal except for the SBR $(\mathrm{Pa} / \mathrm{Ki})$. Of note, no adverse reactions were encountered over the $8 \mathrm{~h}$ observation period. 
A.

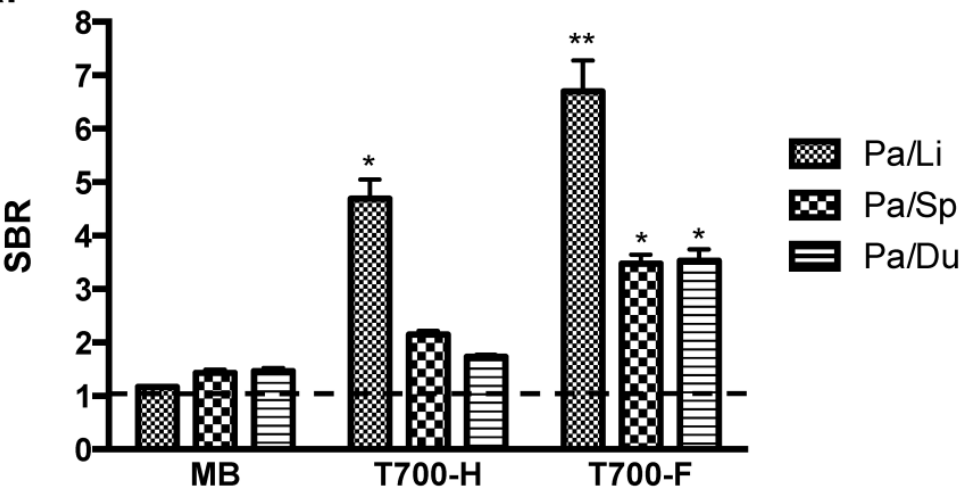

B.

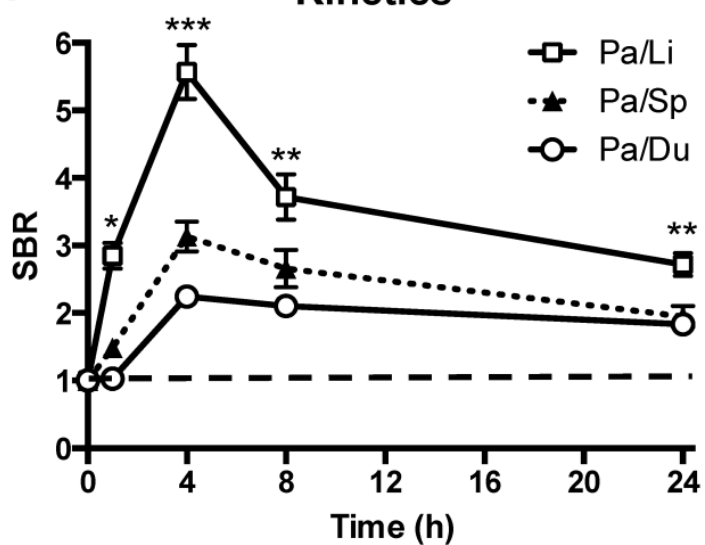

C.

Dose-Dependency

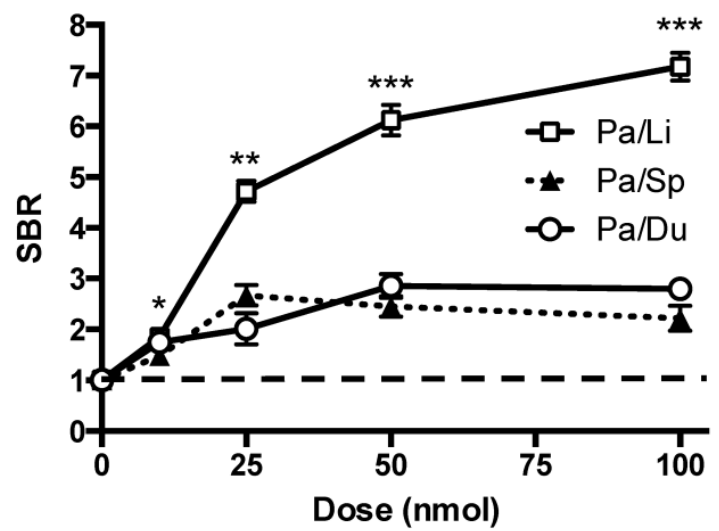

D.

Color Image

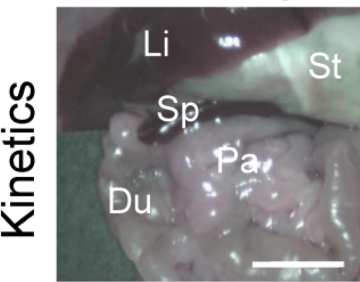

Color Image

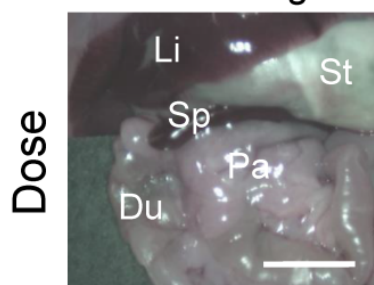

NIR \#1: $0 \mathrm{~h}$

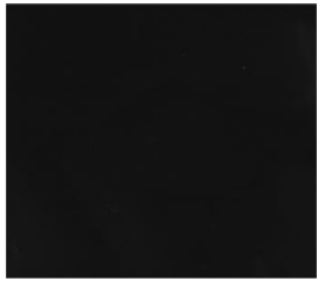

NIR \#1: $0 \mathrm{nmol}$

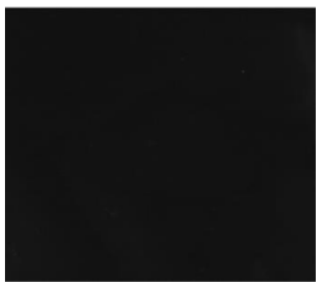

NIR \#1: $1 \mathrm{~h}$

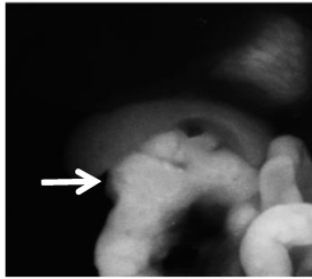

NIR \#1: $10 \mathrm{nmol}$

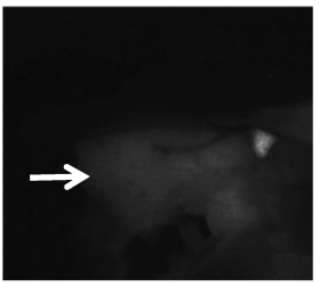

NIR \#1: $4 \mathrm{~h}$

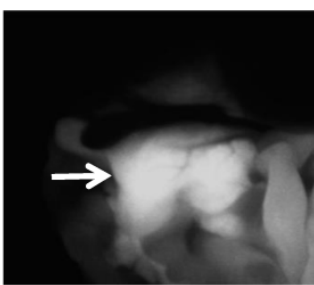

NIR \#1: $25 \mathrm{nmol}$

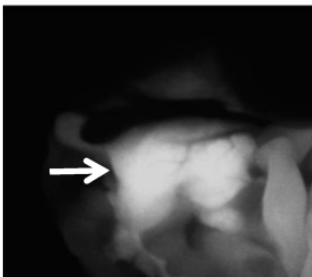

NIR \#1: 8 h

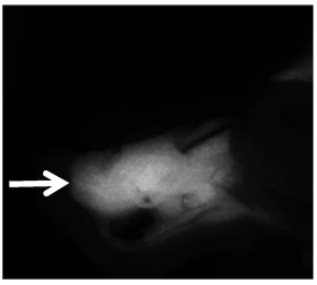

NIR \#1: $50 \mathrm{nmol}$

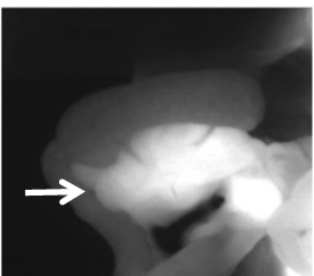

Figure 2. Quantitative Evaluation of NIR Fluorophore Uptake in Mouse Pancreas: a) SBR (mean \pm SD) of pancreas against liver, spleen, and duodenum was compared using various NIR fluorophores. b) Quantitative time-course assessment: $25 \mathrm{nmol}$ of T700-F was injected intravenously into CD-I mice, and SBR (Pa/Li), SBR ( $\mathrm{Pa} / \mathrm{Sp}$ ) and SBR (Pa/Du) were measured at different time points $(T=0, I, 2,4,8$, and $24 \mathrm{~h})$. c) Dose-response curve: $0,10,25,50$ and 100 nmol of T700-F were injected intravenously into CD-I mice, and SBR $(\mathrm{Pa} / \mathrm{Li}), \mathrm{SBR}(\mathrm{Pa} / \mathrm{Sp})$ and SBR $(\mathrm{Pa} / \mathrm{Du})$ were measured at $4 \mathrm{~h}$ post-injection. d) Real-time intraoperative imaging of pancreas in different time and doses. Controls were injected with D5W alone, and at least 3 animals were used for each data point. Scale bars $=0.5 \mathrm{~cm}$. $* P<0.05$, $* * P<0.01$, and $* * * P<0.001$. Abbreviations used are: Du, duodenum; $\mathrm{Li}$, liver; $\mathrm{Pa}$, pancreas (arrow); Sp, spleen; St, stomach; Pa/Li, pancreas-to-liver ratio; Pa/Sp, pancreas-to-spleen ratio; Pa/Du, pancreas-to-duodenum ratio. All NIR fluorescence images have identical exposure times and normalizations. 
A.
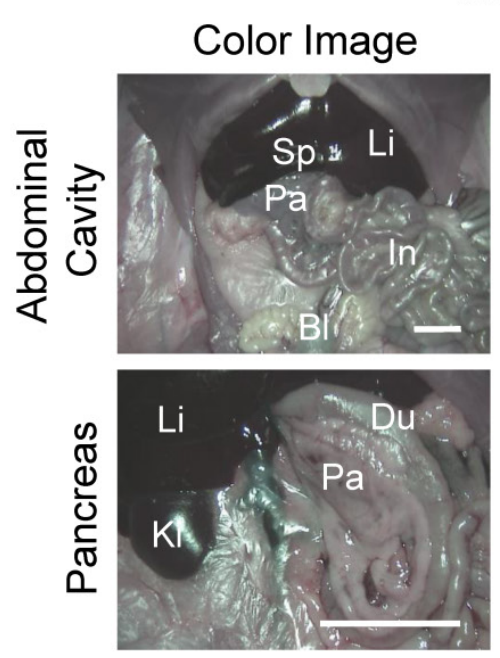

MB
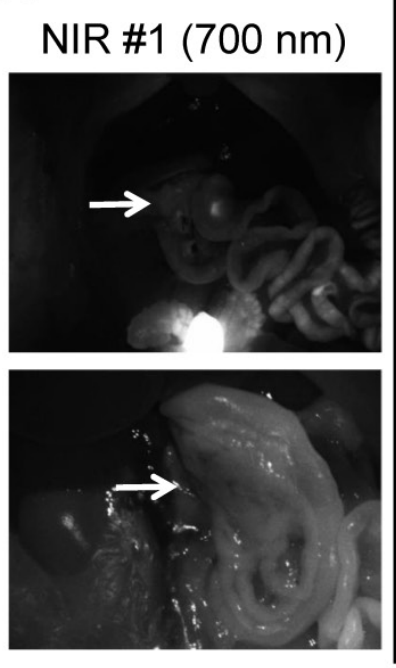
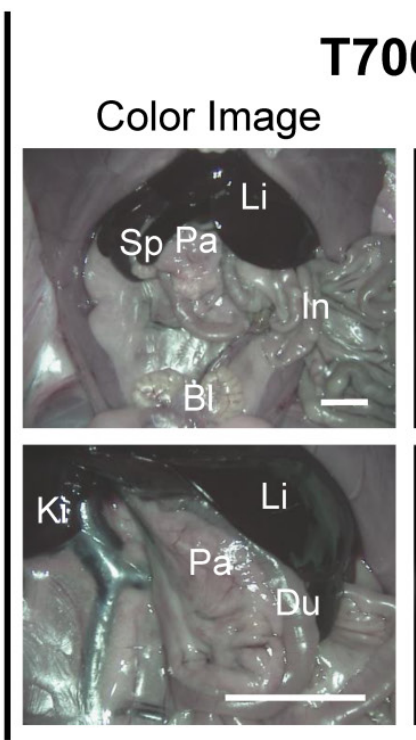

$\mathrm{NIR} \# 1(700 \mathrm{~nm})$
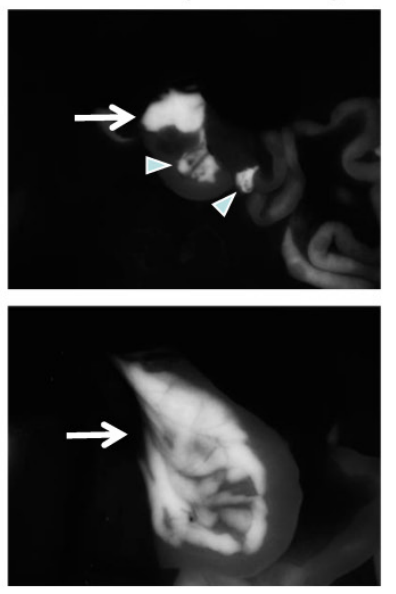

B.
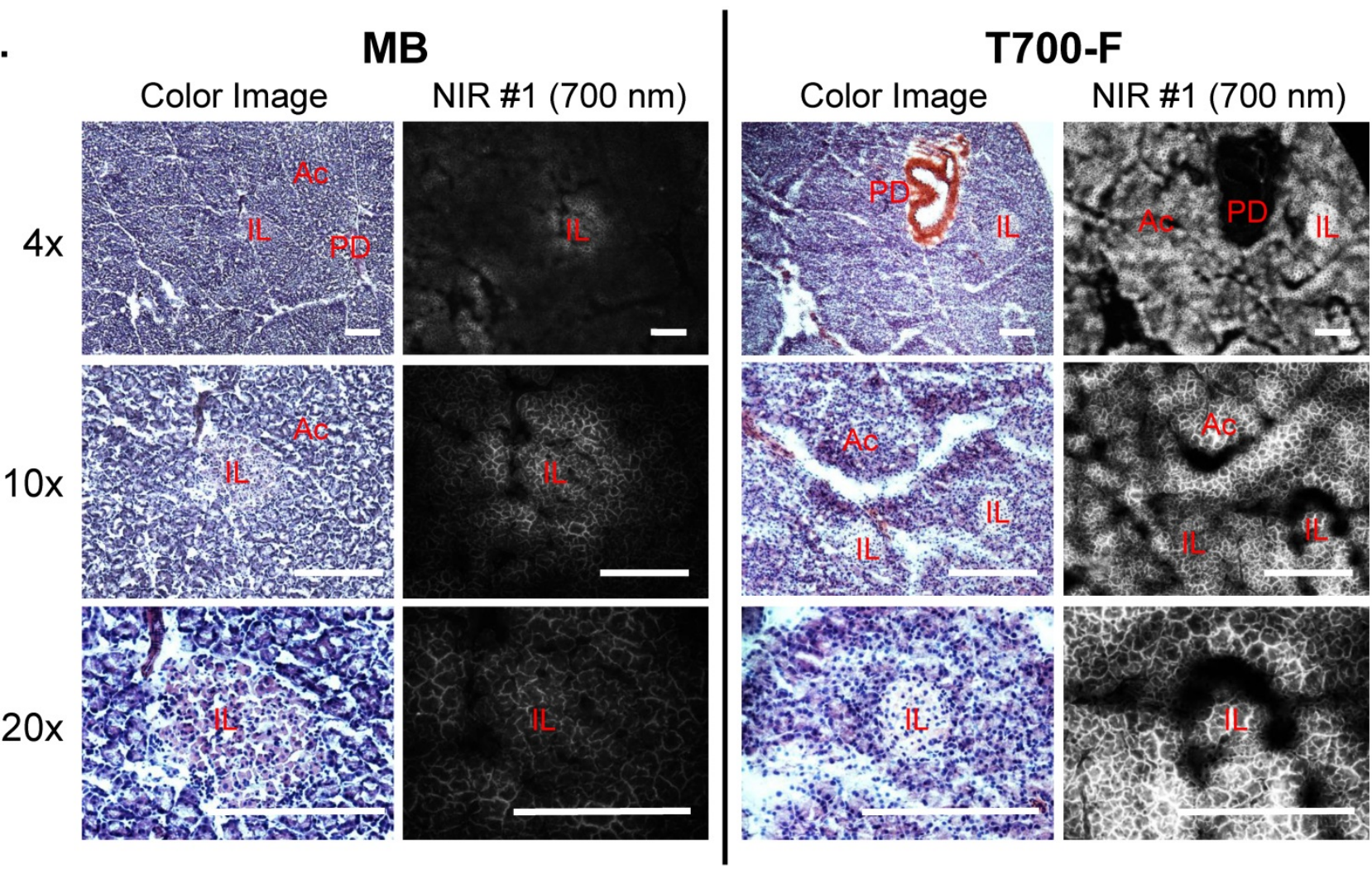

Figure 3. Intraoperative Pancreas Imaging in SD Rats: a) I.2 4 mol of MB and $100 \mathrm{nmol}$ of T700-F were injected intravenously I5 min and 4 h prior to imaging, respectively (n = 3). Abbreviations used are: $\mathrm{Bl}$, bladder; Du, duodenum; In, intestine; Ki, kidney; Li, liver; Pa, pancreas; Sp, spleen. Arrows indicate pancreas and arrowheads indicate autofluorescence of food in lumen of intestine. Scale bars $=1 \mathrm{~cm}$. All NIR fluorescence images have identical exposure times and normalizations. b) Histological analysis: Consecutive sections from rat pancreas stained with H\&E or unstained were observed using an NIR fluorescence microscope. Abbreviations used are: Ac, acinus; IL, islets of Langerhans; PD, pancreatic duct. Scale bars $=300 \mu \mathrm{m}$. All NIR fluorescence images for each condition have identical exposure times and normalizations. 
A.

MB

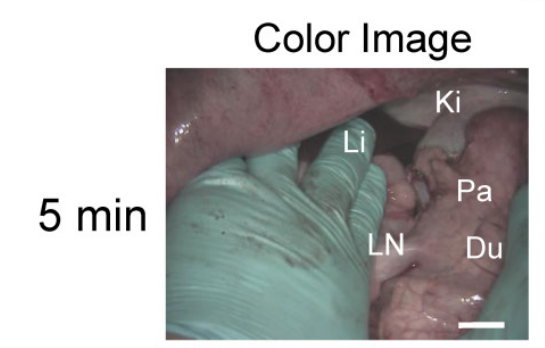

NIR \#1 (700 nm)
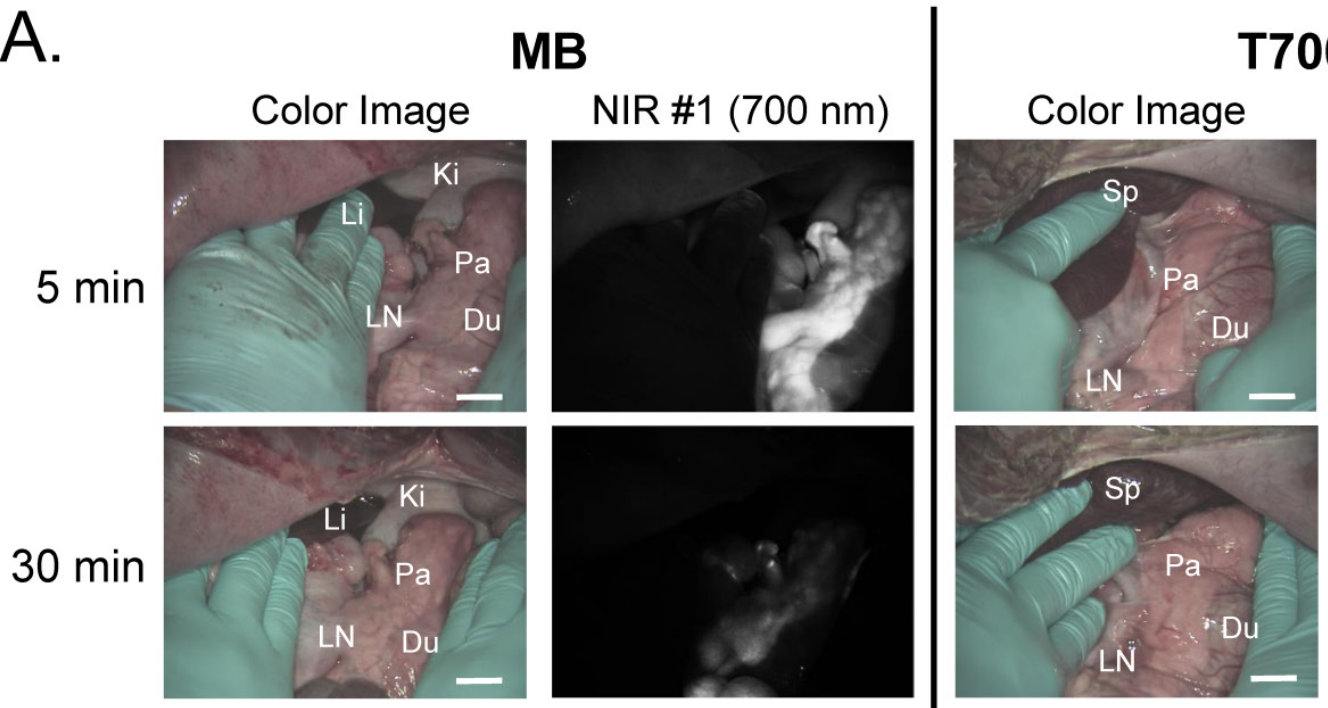

T700-F
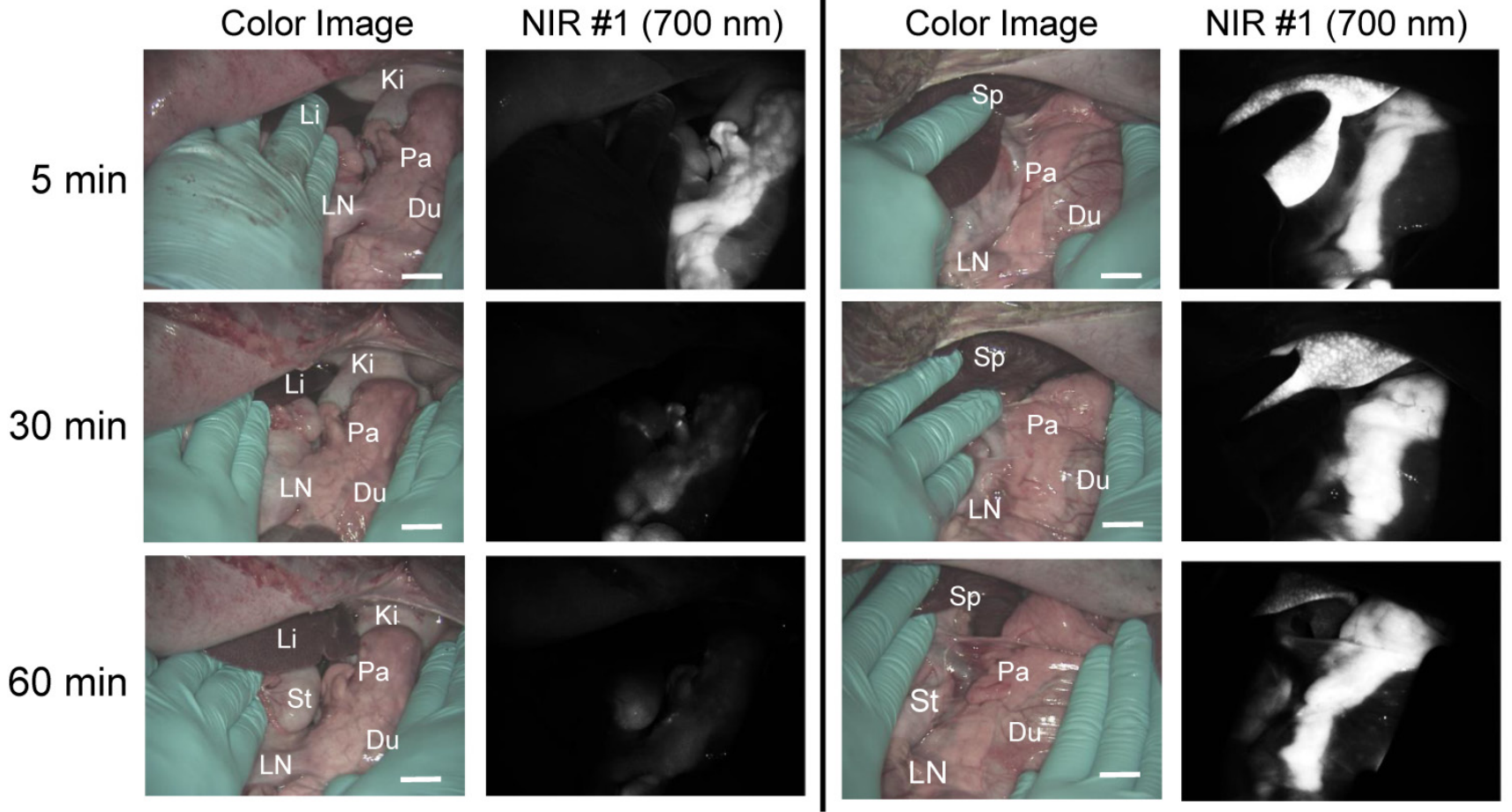

B.

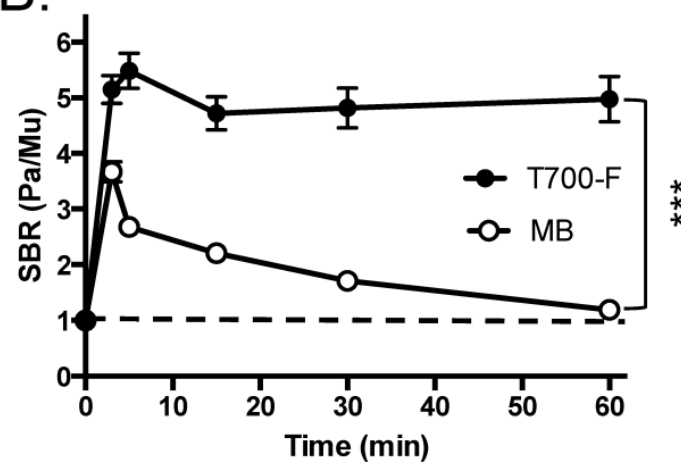

C.
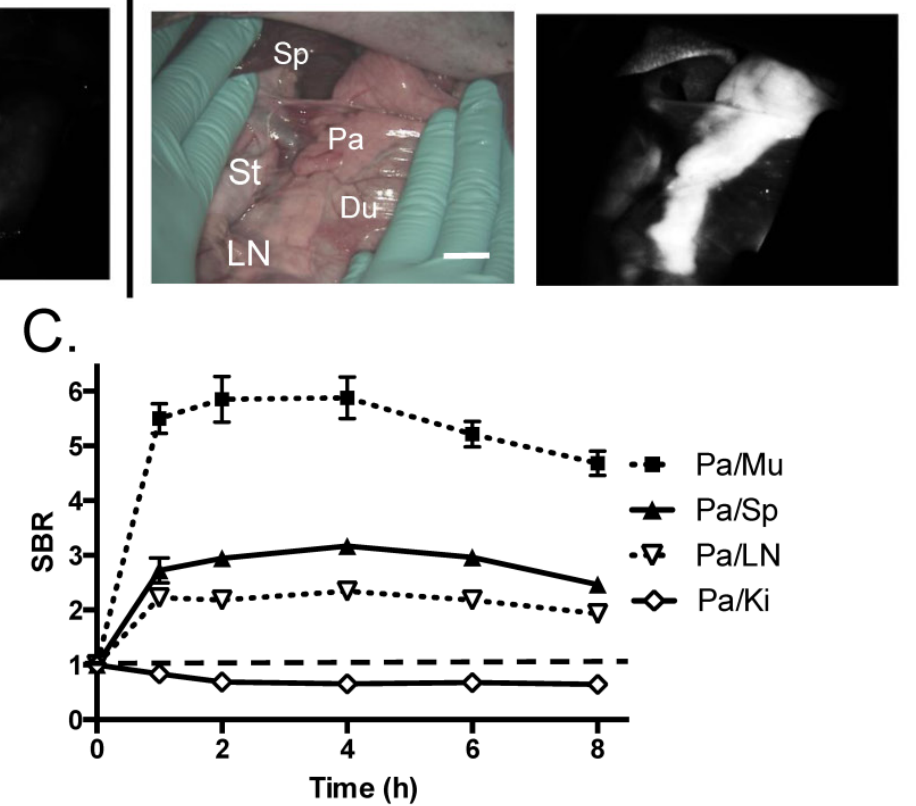

Figure 4. Intraoperative Pancreas Imaging in Pigs: a) $164 \mu \mathrm{mol}$ of MB and $2.5 \mu \mathrm{mol}$ of T700-F were injected intravenously into $35 \mathrm{~kg}$ Yorkshire pigs, and images were recorded over the course of $60 \mathrm{~min}(\mathrm{n}=3)$. Abbreviations used are: Du, duodenum; Ki, kidney; Li, liver; LN, lymph node; Pa, pancreas. All NIR fluorescence images have identical exposure times and normalizations. b) SBR (mean \pm SD) comparison between MB and T700-F: SBR (Pa/Mu) was measured at different time points. ***P < 0.001 . Statistical analysis between $\mathrm{MB}$ and T700-F was performed using unpaired t test. c) Quantitative time-course assessment of SBR (mean \pm SD) for pancreas and surrounding organs/tissues were measured after $2.5 \mu \mathrm{mol}$ of T700-F injection over the course of $8 \mathrm{~h}(\mathrm{n}=3)$. Abbreviations used are: $\mathrm{Pa} / \mathrm{Mu}$, pancreas-to-muscle ratio; $\mathrm{Pa} / \mathrm{Sp}$, pancreas-to-spleen ratio; Pa/LN, pancreas-to-lymph node ratio; $\mathrm{Pa} / \mathrm{Ki}$, pancreas-to-kidney ratio. Scale bars $=1 \mathrm{~cm}$. All NIR fluorescence images have identical exposure times and normalizations.

Simultaneous dual-channel imaging of pancreas for image-guided abdominal surgery. Since the proximity of the pancreas to surrounding tissues and organs, such as arteries, lymph nodes, kidneys, and adrenal glands, is the main cause of intraoperative pancreatic injury, we explored simultaneous dual-channel imaging of pancreas. With the assistance of already developed $800 \mathrm{~nm}$ emitting NIR fluorophores $[13,15,21]$, the FLARE imaging system enabled $700 \mathrm{~nm}$ and $800 \mathrm{~nm}$ NIR fluorescence imaging simultaneously in real time.

To identify blood vessels, ZW800-1 was injected intravenously into pig $4 \mathrm{~h}$ after injection of T700-F. As shown in Figure 5, we could visualize not only im- portant arteries in gastrointestinal surgery such as the common hepatic artery, left gastric artery, and splenic artery, but also extremely small vessel branches from the common hepatic artery.

Because ZW800-1 is cleared from the body via renal excretion, we continuously imaged kidneys and found that kidney signal from ZW800-1 was highest at $2 \mathrm{~h}$ post-injection [21]. As shown in Figure 5, this permitted unambiguous identification of the boundary of pancreas from the kidney.

In subsequent experiments, we imaged peripancreatic lymph nodes by injecting ZW800-3C along with T700-F $4 \mathrm{~h}$ prior to imaging [13]. Lymph nodes were clearly visualized even when obscured by over- 
lying pancreatic tissue, with the color merged image showing all desired surgical landmarks on one screen.

Finally, for dual-channel imaging of pancreas and adrenal glands, T700-F was injected $4 \mathrm{~h}$ prior to imaging, followed by injecting ESNF31 intravenously in the same pig $30 \mathrm{~min}$ prior to imaging [15]. Pancreas and adrenal glands were readily imaged using the 700 $\mathrm{nm}$ and $800 \mathrm{~nm}$ NIR channels, respectively. This dual-channel imaging permitted the pancreas to be distinguished from peripancreatic lymph nodes and the adrenal gland rather easily.

\section{Discussion}

Recent technical advances in abdominal surgery have made complex procedures safer and shorter. However, pancreas-related complications after gas- trectomy with radical lymphadenectomy including peripancreatic lymph nodes resection, splenectomy, adrenalectomy, and nephrectomy remain unacceptably high $[1,2,9,10]$. Even with a magnified view provided by laparoscopy, it remains difficult to avoid pancreatic injury because of relatively poor contrast between pancreas and surrounding tissues and organs, and the inability of visible light to penetrate through blood and overlying tissue. NIR light has the potential to solve this problem because it can penetrate 5-8 mm into living tissue [28] and specific NIR contrast agents can be used to highlight specific anatomy. Importantly, NIR fluorescence imaging systems are now widely available for both open and minimally-invasive surgery.
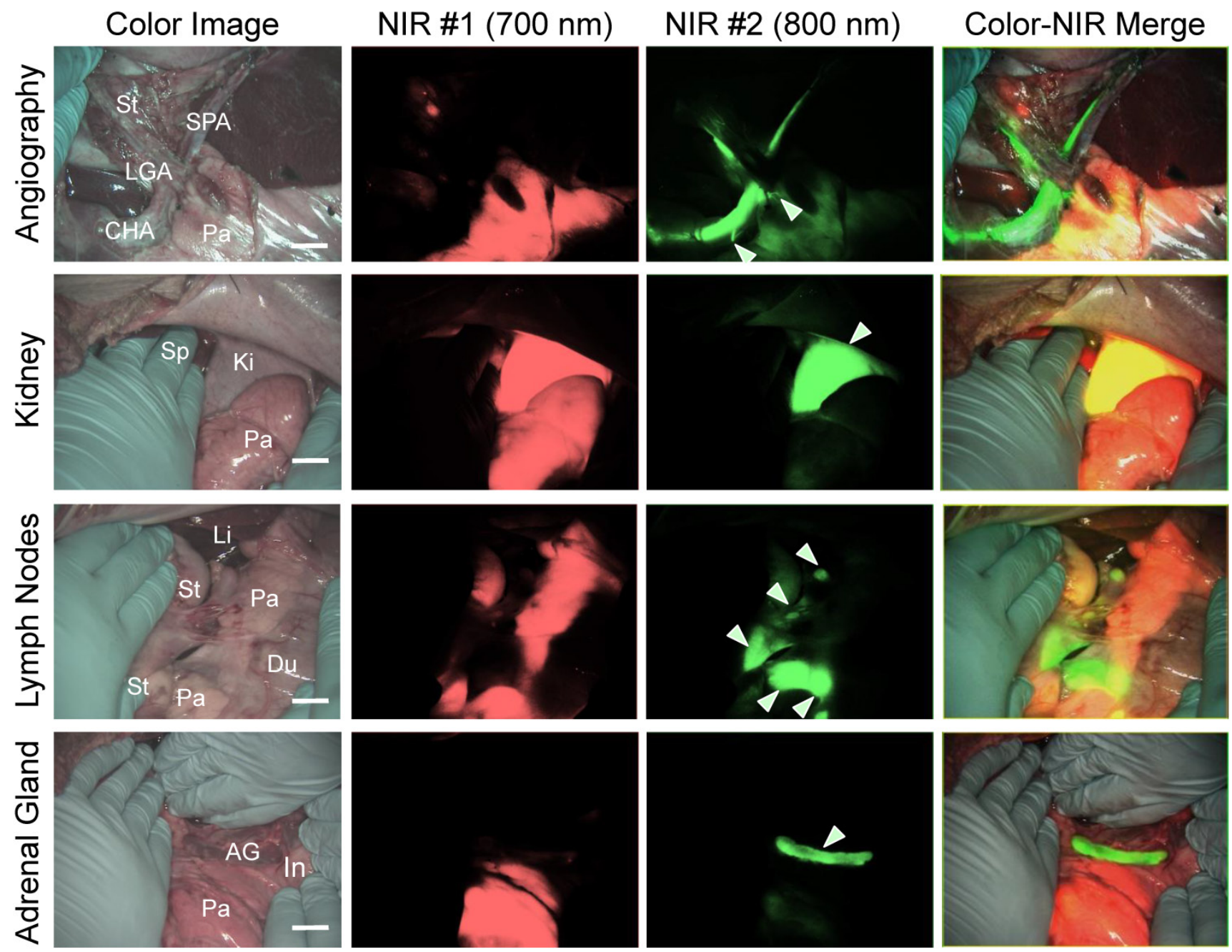

Figure 5. Simultaneous Dual Channel Imaging of Pancreas and Surrounding Tissues and Organs: $2.5 \mu$ mol of T700-F was injected intravenously for pancreas imaging $4 \mathrm{~h}$ prior to: I st row: NIR angiography with $2 \mu \mathrm{mol}$ intravenous injection of ZW800-I immediately prior to imaging. 2nd row: Kidney imaging with $2 \mu$ mol intravenous injection of ZW800-I 30 min prior to imaging. $3^{\text {rd }}$ row: Pan lymph node imaging with I $\mu$ mol intravenous injection of ZW800-3C $4 \mathrm{~h}$ prior to imaging. $4^{\text {th }}$ row: Adrenal gland imaging with $2 \mu$ mol intravenous injection of ESNF3I 30 min prior to imaging ( $\mathrm{n}=3$ pigs). Shown are color image, $700 \mathrm{~nm}$ NIR fluorescence, $800 \mathrm{~nm}$ NIR fluorescence, and a merged image of the three. For the merged image, FLARE TM channel \#I (700 nm) is pseudo-colored in red and channel \#2 (800 nm) in green. Abbreviations used are: AG, adrenal gland; CHA, common hepatic artery; Du, duodenum; In, intestine; Ki, kidney; Li, liver; LGA, left gastric artery; Pa, pancreas; Sp, spleen; St, stomach; SPA, splenic artery. Arrowheads indicate tiny branch from artery (I st row), kidney (2nd row), pan lymph nodes ( $3^{\text {rd }}$ row), or adrenal gland (4th row). Scale bars $=I \mathrm{~cm}$. All NIR fluorescence images have identical exposure times and normalizations. 
MB is a NIR fluorophore that we previously demonstrated can highlight the pancreas, and interestingly, insulinoma, however, it has relatively poor optical properties and short retention in the pancreas [11]. Out of over 300 candidates, T700-F showed significantly high uptake in pancreas and low background tissue uptake. Furthermore, its retention in pancreas was over $8 \mathrm{~h}$, making this agent potentially useful in complex abdominal surgeries including laparoscopic operations (Figure 4C) [29]. Although the mechanism of specific pancreas uptake and retention of T700-F is currently unknown, it appears that the physicochemical properties of the substituted pentamethine play a key role for targeting. T700-F differs from T700-H by only 2 fluorine atoms, yet these atoms result in significantly improved pancreas selectivity.

There are some limitations, though, to the present study. First, our prototype pancreas-targeted compound T700-F emits at $700 \mathrm{~nm}$ where autofluorescence exists from fecal contents. A thorough bowel cleansing is therefore important. Development of an $800 \mathrm{~nm}$ version, which would solve this problem, is ongoing. Second, T700-F shows nonspecific uptake in kidneys, spleen, and lymph nodes and kidney, especially, has a higher signal than pancreas. However, as shown in Figure 5, this limitation is easily overcome by a low-dose injection of ZW800-1, which permits unambiguous separation of kidney from pancreas. Because first-in-human trials of ZW800-1 are imminent, it should be available for this application in the near future. Third, T700-F will require the same regulatory path as any drug prior to human use. Despite these limitations, this study lays the foundation for real-time intraoperative pancreas imaging during complex abdominal surgeries.

\section{Supplementary Material}

Fig.S1. http:/ / www.thno.org/v05p0001s1.pdf

\section{Abbreviations}

NIR: near-infrared; MB: methylene blue; DMSO: dimethyl sulfoxide; FBS: fetal bovine serum; QY: quantum yield; ICG: indocyanine green; AAALAC: association for assessment and accreditation of laboratory animal care; IACUC: institutional animal care and use committee; FOV: field of view; D5W: 5\% dextrose in water; SBR: signal-to-background ratio; PLN: pan lymph node; FI: fluorescent intensity; ROI: region of interest; $\mathrm{BG}$ : background; $\mathrm{Pa} / \mathrm{Mu}$ : pancreas-to-muscle ratio; $\mathrm{Pa} / \mathrm{Li}$ : pancreas-to-liver ratio; $\mathrm{Pa} / \mathrm{Sp}$ : pancreas-to-spleen ratio; $\mathrm{Pa} / \mathrm{Du}$ : pancreas-to-duodenum ratio; $\mathrm{Pa} / \mathrm{Ln}$ : pancreas-to-lymph node ratio; $\mathrm{Pa} / \mathrm{Ki}$ : pancreas-to-kidney ratio; $\mathrm{SD}$ : standard deviation; AC: acini; IL: islets of Langerhans;
Pa: pancreas; Mu: muscle; Sp: spleen; Ln: lymph nodes; Ki: kidneys; MW: molecular weight; Abs: absorbance; FL: fluorescence; Du: duodenum; Li: liver; St: stomach; SD: Sprague-Dawley; Bl: bladder; In: intestine; PD: pancreatic duct; $\mathrm{Pb}$ : pancreas body; $\mathrm{Ph}$ : pancreas head; Pt: pancreas tail; AG: adrenal gland; CHA: common hepatic artery; LGA: left gastric artery; SPA: splenic artery.

\section{Acknowledgments}

We thank Rita G. Laurence for assistance with animal surgery, David Burrington, Jr. for editing, and Eugenia Trabucchi for administrative assistance, and Frank Kettenring and Florin Neacsu for assistance with development and maintenance of the FLARE imaging system and software.

\section{Funding and all other required statements}

This study was supported by the following grants from the National Institutes of Health: NCI BRP grant \#R01-CA-115296 (JVF), NIBIB grant \#R01-EB-011523 (HSC), and a grant from the Dana Foundation (HSC). This content is solely the responsibility of the authors and does not necessarily represent the official views of the National Institutes of Health.

\section{Author's Contributions}

HW, JVF, MH and HSC designed the study. HW, $\mathrm{HH}, \mathrm{CV}$, JG, GP and MH performed the experiments. HW, SG, JVF, MH and HSC reviewed, analyzed, and interpreted the data. HW, JVF, MH and HSC wrote and revised the paper. All authors discussed the results and commented on the manuscript. JVF, MH and HSC approved the submitted version.

**This research and paper is not presented at any communications or meetings.

\section{Competing Interests}

John V. Frangioni, M.D., Ph.D.: Dr. Frangioni is currently CEO of Curadel, LLC, which has licensed FLARE imaging systems and contrast agents from the Beth Israel Deaconess Medical Center.

\section{References}

1. Obama K, Okabe H, Hosogi H, Tanaka E, Itami A, Sakai Y. Feasibility of laparoscopic gastrectomy with radical lymph node dissection for gastric cancer: from a viewpoint of pancreas-related complications. Surgery. 2011; 149: $15-21$

2. Jiang X, Hiki N, Nunobe S, Kumagai K, Nohara K, Sano T, et al. Postoperative pancreatic fistula and the risk factors of laparoscopy-assisted distal gastrectomy for early gastric cancer. Ann Surg Oncol. 2012; 19: 115-21.

3. Nobuoka D, Gotohda N, Konishi M, Nakagohri T, Takahashi S, Kinoshita T. Prevention of postoperative pancreatic fistula after total gastrectomy. World J Surg. 2008; 32: 2261-6.

4. Ichikawa D, Kurioka H, Yamaguchi T, Koike H, Okamoto K, Otsuji E, et al. Postoperative complications following gastrectomy for gastric cancer during the last decade. Hepatogastroenterology. 2004; 51: 613-7.

5. Okabayashi T, Kobayashi M, Sugimoto T, Okamoto K, Matsuura K, Araki K. Postoperative pancreatic fistula following surgery for gastric and pancreatic 
neoplasm; is distal pancreaticosplenectomy truly safe? Hepatogastroenterology. 2005; 52: 233-6.

6. Kunisaki C, Shimada H, Ono H, Otsuka Y, Matsuda G, Nomura M, et al. Predictive factors for pancreatic fistula after pancreaticosplenectomy for advanced gastric cancer in the upper third of the stomach. J Gastrointest Surg. 2006; 10: 132-7.

7. Tanaka K, Miyashiro I, Yano M, Kishi K, Motoori M, Seki Y, et al. Accumulation of excess visceral fat is a risk factor for pancreatic fistula formation after total gastrectomy. Ann Surg Oncol. 2009; 16: 1520-5.

8. Miki Y, Tokunaga M, Bando E, Tanizawa Y, Kawamura T, Terashima M. Evaluation of postoperative pancreatic fistula after total gastrectomy with D2 lymphadenectomy by ISGPF classification. J Gastrointest Surg. 2011; 15: 1969-76.

9. Wang X, Li Y, Crook N, Peng B, Niu T. Laparoscopic splenectomy: a surgeon's experience of 302 patients with analysis of postoperative complications. Surg Endosc. 2013; 27: 3564-71.

10. Varkarakis IM, Allaf ME, Bhayani SB, Inagaki T, Su LM, Kavoussi LR, et al. Pancreatic injuries during laparoscopic urologic surgery. Urology. 2004; 64: 1089-93.

11. Winer JH, Choi HS, Gibbs-Strauss SL, Ashitate Y, Colson YL, Frangioni JV. Intraoperative localization of insulinoma and normal pancreas using invisible near-infrared fluorescent light. Ann Surg Oncol. 2010; 17: 1094-100.

12. Hyun H, Park MH, Owens EA, Wada H, Henary M, Frangioni JV, et al. Structure-inherent targeting of near-infrared fluorophores: parathyroid and thyroid gland imaging using fluorinated polymethines. Nat Med. 2014: in press.

13. Ashitate $\mathrm{Y} \mathrm{HH}$, Kim SH, Lee JH, Henary M, Frangioni JV, Choi HS. . Simultaneous Mapping of Pan and Sentinel Lymph Nodes for Real-Time Image-Guided Surgery. Theranostics. 2014; 4: 693-700.

14. Park MH, Hyun $H$, Ashitate $Y$, Wada H, Park G, Lee JH, et al. Prototype nerve-specific near-infrared fluorophores. Theranostics. 2014; 4: 823-33.

15. Ashitate Y, Park MH, Hyun H, Venugopal V, Lee JH, Park G, et al. Near-infrared fluorophores for dual-channel image-guided adrenal gland surgery. In review.

16. Hyun H, Wada H, Henary M, Frangioni JV, Choi HS. Direct visualization of phosphonated contrast agent uptake in bone. Angew Chem Int Ed Engl. 2014; in press.

17. Troyan SL, Kianzad V, Gibbs-Strauss SL, Gioux S, Matsui A, Oketokoun R, et al. The FLARE intraoperative near-infrared fluorescence imaging system: a first-in-human clinical trial in breast cancer sentinel lymph node mapping. Ann Surg Oncol. 2009; 16: 2943-52.

18. Kim SH, Park G, Hyun H, Lee JH, Ashitate $Y$, Choi J, et al. Near-infrared lipophilic fluorophores for tracing tissue growth. Biomed Mater. 2013; 8: 014110 .

19. Lee JH, Hyun H, Cross CJ, Henary M, Nasr KA, Oketokoun R, et al. Rapid and Facile Microwave-Assisted Surface Chemistry for Functionalized Microarray Slides. Adv Funct Mater. 2012; 22: 872-8.

20. Lee JH, Park S, Hyun $H$, Bordo MW, Oketokoun $R$, Nasr KA, et al. High-throughput screening of small molecule ligands targeted to live bacteria surface. Anal Chem. 2013; 85: 3508-14

21. Choi HS, Nasr K, Alyabyev S, Feith D, Lee JH, Kim SH, et al. Synthesis and in vivo fate of zwitterionic near-infrared fluorophores. Angew Chem Int Ed Engl. 2011; 50: 6258-63.

22. Choi HS, Ashitate Y, Lee JH, Kim SH, Matsui A, Insin N, et al. Rapid translocation of nanoparticles from the lung airspaces to the body. Nat Biotechnol. 2010; 28: 1300-3.

23. Ashitate Y, Tanaka E, Stockdale A, Choi HS, Frangioni JV. Near-infrared fluorescence imaging of thoracic duct anatomy and function in open surgery and video-assisted thoracic surgery. J Thorac Cardiovasc Surg. 2011; 142: 31-8.

24. Gioux S, Choi HS, Frangioni JV. Image-guided surgery using invisible near-infrared light: fundamentals of clinical translation. Mol Imaging. 2010; 9: 237-55.

25. Ashitate Y, Stockdale A, Choi HS, Laurence RG, Frangioni JV. Real-time simultaneous near-infrared fluorescence imaging of bile duct and arterial anatomy. J Surg Res. 2012; 176: 7-13.

26. Nakayama A, Bianco AC, Zhang CY, Lowell BB, Frangioni JV. Quantitation of brown adipose tissue perfusion in transgenic mice using near-infrared fluorescence imaging. Mol Imaging. 2003; 2: 37-49.

27. Gibbs-Strauss SL, Nasr KA, Fish KM, Khullar O, Ashitate Y, Siclovan TM, et al, Nerve-highlighting fluorescent contrast agents for image-guided surgery. Mol Imaging. 2011; 10: 91-101.

28. Vahrmeijer AL, Hutteman M, van der Vorst JR, van de Velde CJ, Frangioni JV. Image-guided cancer surgery using near-infrared fluorescence. Nat Rev Clin Oncol. 2013; 10: 507-18.

29. Venugopal V, Park M, Ashitate Y, Neacsu F, Kettenring F, Frangioni JV, et al. Design and characterization of an optimized simultaneous color and near-infrared fluorescence rigid endoscopic imaging system. J Biomed Opt. 2013; 18: 126018. 